\title{
Collaborative multi-agent reinforcement learning based on experience propagation
}

\author{
Min Fang ${ }^{1, *}$ and Frans C.A. Groen ${ }^{2}$ \\ 1. School of Computer Science and Technology, Xidian University, Xi' an 710071, China; \\ 2. Informatics Institute, University of Amsterdam, Amsterdam 1098 XH, Netherlands
}

\begin{abstract}
For multi-agent reinforcement learning in Markov games, knowledge extraction and sharing are key research problems. State list extracting means to calculate the optimal shared state path from state trajectories with cycles. A state list extracting algorithm checks cyclic state lists of a current state in the state trajectory, condensing the optimal action set of the current state. By reinforcing the optimal action selected, the action policy of cyclic states is optimized gradually. The state list extracting is repeatedly learned and used as the experience knowledge which is shared by teams. Agents speed up the rate of convergence by experience sharing. Competition games of preys and predators are used for the experiments. The results of experiments prove that the proposed algorithms overcome the lack of experience in the initial stage, speed up learning and improve the performance.
\end{abstract}

Keywords: multi-agent, $Q$ learning, state list extracting, experience sharing.

DOI: 10.1109/JSEE.2013.00079

\section{Introduction}

A primary problem is how to adopt the single agent's actions to the dynamic environment to improve the system performance. Multi-agent reinforcement learning was used to solve multi-agent's coordination and cooperation [1-3]. Most of the multi-agent reinforcement learning algorithms assume that an agent knows the game structure [4-6], or the Nash Equilibrium. Some algorithms need to know what actions other agents selected and what rewards they received. In multi-agent systems, each agent gets instant rewards according not only to its own actions, but also to the actions of cooperative agents [7-10]. Therefore, if we formalize each discrete state into a strategy, then the Markov decision process (MDP) model of reinforcement learning can be viewed as a multi-agent Markov game model. Most of the discussions of reinforcement learning

Manuscript received July 01, 2012.

*Corresponding author.

This work was supported by the National Natural Science Foundation of China (61070143; 61173088). in dynamic multi-agent environments are based on a Markov game, which is also called a Stochastic game. Under the nonzero-sum strategy model, an agent hopes to get an optimal solution by refining its own policy and analyzing its competitors' policy. The exploration-exploitation tradeoff problem is always important for reinforcement learning. Both the $\varepsilon$-greedy strategy and the Boltzman exploration strategy are common strategies for agents to explore the current knowledge. In the beginning of the learning process, an agent has zero knowledge of the environment, and the experiences of an agent are very limited, so the $Q$ value cannot accurately expresses the environment [11]. By adopting the exploratory action selection policy, the agent's exploration ability has to be improved step by step. Therefore, an agent may often visit the same state many times before it reaches the goal during learning. The larger the state space of agents is, the harder the action calculation is.

One of the fundamental problems for cooperative multiagent is how to provide an interaction way for knowledge exchange among agents $[4,12,13]$. The state trajectory of an agent transferring from the initial state to the current state is recorded in a state list set. Multiple agents operate separately in multiple independent processes in the same state space. How can they share the common knowledge? We propose an optimization method of cyclic state paths to the end. This method can extract optimal paths from cyclic state lists as experience knowledge, which can be shared among agents or teams for speeding up the convergence of the value function.

The rest of this paper is organized as follows. We analyze the shared knowledge of state lists in Section 2. Section 3 gives an algorithm for state lists extraction. A new method of experience propagation based on optimal action sets is presented in Section 4. Reinforcement learning using experience sharing is given in Section 5. Section 6 designs games to illustrate this approach. Section 7 provides the conclusions. 


\section{Analyses of state lists}

\subsection{State lists}

A state list $\left(s_{i}, s\right)$ denotes a state sequence from $s_{i}$ to $s$, and $s$ is a goal state. Every state reaching $s$ is added to a list in order. A state $s_{i}$ may appear several times in a list leading to a state $s$ shown as Fig. 1 .

$$
s_{i}^{t} \longrightarrow s_{m} \longrightarrow s_{n} \longrightarrow s_{i}^{t+k} \longrightarrow s_{p} \longrightarrow s
$$

Fig. 1 State $s_{i}$ to state $s$

In Fig. $1, s_{i}^{t}$ and $s_{i}^{t+k}$ denote the state $s_{i}$ at two different time steps $t$ and $t+k$, respectively. The next state of $s_{i}^{t+k}$ is different from that of $s_{i}^{t}$ by selecting a different action, resulting in a different state. The former is $s_{p}$, and the latter is $s_{m}$. Compare the $Q$ values of the state $s_{i}$ at different time steps $t$ and $t+k$. If $\boldsymbol{Q}\left(s_{i}^{t}, a^{t}\right)<\boldsymbol{Q}\left(s_{i}^{t+k}, a^{t+k}\right)$, there is a better action selecting possible in state $s_{i}^{t}$ which can transform to the state $s_{p}$. We can connect state $s_{i}^{t}$ to $s_{p}, s_{i}^{t} \rightarrow s_{p}$, and extract state lists as the experience of action selections. The transformation of the states is shown in Fig. 2.

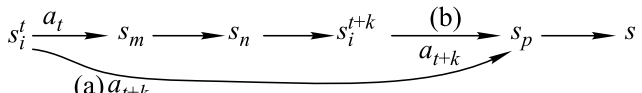

Fig. 2 State list

These state lists extracted according to experiences are saved in a dataset and can be shared among the agents. The state lists of the state $s$ are shown as Fig. 3 .

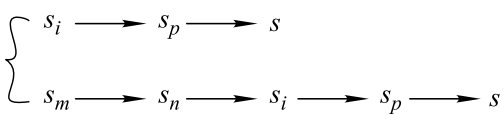

Fig. 3 State lists of state $s$

From Fig. 2, after the state $s_{i}$ is visited at time step $t$, with extracted experience, no updates for $\left(s_{i}, a_{t+k}\right)$ occur at time steps $t$ to $t+k$, so

$$
\boldsymbol{Q}_{t+1}\left(s_{i}, a_{t+k}\right)=\boldsymbol{Q}_{t}\left(s_{i}, a_{t+k}\right) .
$$

We use $\widetilde{Q}$ to denote the $Q$ value by extracted experience in this section.

Theorem 1 Assume a state transition sequence of an agent as shown in Fig. 2, and actions $a^{t}, a^{t+k}$ as two actions at state $s_{i}$. For the reinforcement learning with experience sharing, the policy $\pi$ is set as $\pi\left(s_{i}\right)=a^{t+k}$.

Proof According to (1) and the step (b) of Fig. 2,

$$
\begin{gathered}
\boldsymbol{Q}_{t+(k+1)}\left(s_{i}, a_{t+k}\right)= \\
(1-\alpha) \boldsymbol{Q}_{t+k}\left(s_{i}, a_{t+k}\right)+\alpha\left[r_{t+1}+\gamma \max _{a^{\prime}} \boldsymbol{Q}_{t+1}\left(s_{p}, a^{\prime}\right)\right] .
\end{gathered}
$$

From the step (a) of Fig. 2, then

$$
\begin{gathered}
\widetilde{\boldsymbol{Q}}_{t+1}\left(s_{i}, a_{t+k}\right)= \\
(1-\alpha) \widetilde{\boldsymbol{Q}}_{t}\left(s_{i}, a_{t+k}\right)+\alpha\left[r_{t}+\gamma \max _{a^{\prime}} \widetilde{\boldsymbol{Q}}_{t}\left(s_{p}, a^{\prime}\right)\right] .
\end{gathered}
$$

No updates for $\left(s_{i}, a_{t+k}\right)$ occur from time steps $t$ to $t+k$, so

$$
\boldsymbol{Q}_{t+k}\left(s_{i}, a_{t+k}\right)=\widetilde{\boldsymbol{Q}}_{t}\left(s_{i}, a_{t+k}\right) .
$$

Since (2) holds, we can get

$$
\widetilde{\boldsymbol{Q}}_{t+1}\left(s_{i}, a_{t+k}\right)=\boldsymbol{Q}_{t+(k+1)}\left(s_{i}, a_{t+k}\right) .
$$

For a state $s_{i}$, the condition of extracted experience is

$$
\boldsymbol{Q}_{t+(k+1)}\left(s_{i}, a_{t+k}\right)>\boldsymbol{Q}_{t+1}\left(s_{i}, a_{t+k}\right) .
$$

Since (5) and (6) hold, we can get

$$
\widetilde{\boldsymbol{Q}}_{t+1}\left(s_{i}, a_{t+k}\right)>\boldsymbol{Q}_{t+1}\left(s_{i}, a_{k}\right) .
$$

This indicates that selecting the action $a_{t+k}$ can get a larger reward than selecting the action $a_{t}$ at the state $s_{i}$. According to this experience, the state $s_{p}$ should be the next state of $s_{i}$ instead of the state $s_{m}$.

It means that transferring directly from the state $s_{i}$ to $s_{p}$ can be shared by the other agents in a team. Optimizing $Q$ learning which extracts state lists according to the experience sharing does not cause the failure of the optimal policy.

\subsection{Shared state lists}

For cooperative multi-agent reinforcement learning, a fundamental and important problem is to provide an interaction medium for knowledge exchange and sharing among agents [14]. It is very important to extract state list knowledge and exchange and share this knowledge among cooperative teams in the Markov game.

Many algorithms can be used to refine [15] or aggregate recurrent states based on models. The self-adaptive clustering strategy [16] clusters states according to the iteration results of Bellman. The method has unique iteration and tolerance properties in the state aggregation. The hierarchical reinforcement learning (HRL) algorithm proposed by Dietterich [17] can aggregate states in subtasks if all the strategies are treated in the same way. The similar $Q$ values of an action would be aggregated if and only if states have the same optimal action set, and the aggregation is allowed to take place during the system's MDP selflearning process [18-20]. The online aggregation strategy aggregates the states with the same optimal action. However, this MDP learning process may not create an optimal strategy, which may lead to non-convergence.

We present an algorithm for state list extracting based on the state trajectory. It tracks the state trajectory and finds 
repeated state sequences. The value function updating of repeated states can propagate to every state in its partial state trajectory along the state path between them.

\section{Extracting shared state lists}

Here are the definitions of some symbols:

ListBase 1 is a state list set, composed of state lists.

ListBase 2 is composed of state lists which can be shared by agents of all teams.

trajectory_list is a state trajectory of an agent.

state list is an extracted state list which is being built. It is an acyclic list which can be obtained by removing a cycle from the trajectory_list and will be added to the ListBase 1 .

Each state list in ListBase 1 is an acyclic shortest state trajectory. Agents can share state space knowledge in the ListBase 1 with one another. Shared lists which do not appear in the ListBase 1 join ListBase2. That is a set of shared state lists. How can we discard those repeated sub-lists in the trajectory_list to obtain a set of shared state lists? We present a framework of state lists extracted and shared in Fig. 4.

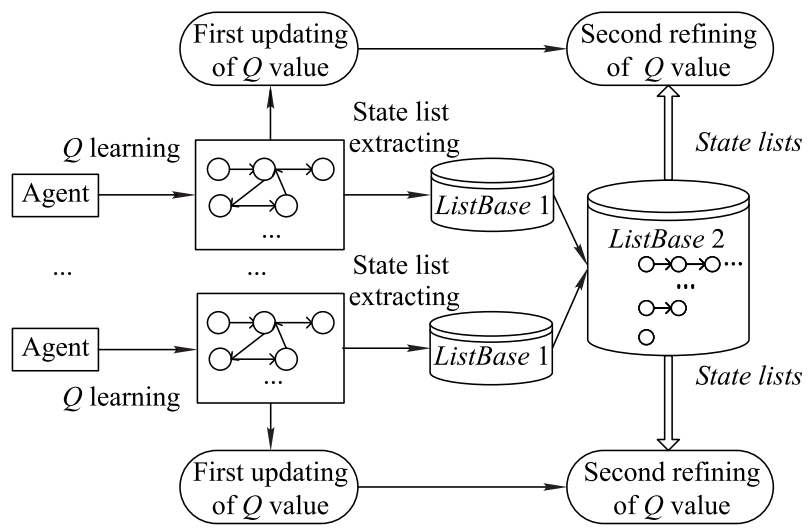

Fig. 4 Frame of state list extracted and shared

When the domain has a large state space and many actions that an agent can choose, the number of possible state transitions becomes large. Because there is not enough experience and knowledge that can be used at the beginning of the learning, some state-action transitions may be visited repeatedly. These states visited repeatedly compose some cyclic paths of states which often exist during the $Q$-learning process. We study an algorithm of state list extracting to find repeated state sequences from one state to another without sacrificing the learning ability of tasks. We construct a set of state lists ListBase 2 as shared knowledge from the start state to the end state in learning iterations.

Each agent creates a state trajectory which includes all the states that the agent visited. Fig. 5 shows this procedure, where the state $s_{0}$ is an initial state and $s_{i}$ is the current state.

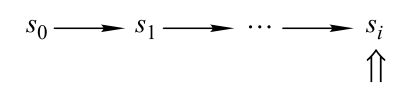

Fig. 5 State list of an agent

For the current state $s_{i}$, if it does not exist in any list of ListBase $1, s_{i}$ is added into the extracted list state_list. If the current state $s_{i}$ already exists in one or some lists of ListBase 1, it means this state has been visited in the past, and if the current state $s_{i}$ is inserted in current position, a cyclic path will be created.

The process of handing a current state $s_{i}$ which has existed in an extracted list is shown in Fig. 6. The process of handing the current state $s_{i}$ which has existed in one list of the shared list set is described in Fig. 7. We give two examples which show the extracting process of shared state lists. The process of state lists $s_{a} \rightarrow s_{b} \rightarrow s_{c} \rightarrow s_{a} \rightarrow$ $s_{d} \rightarrow s_{f}$ and $s_{a} \rightarrow s_{b} \rightarrow s_{c} \rightarrow s_{f}$ are given in Fig. 6(a) and Fig. 7(a), respectively. The changes of an extracted list are given from the step (1) to step (4) in Figs. 6 and 7.

(i) The inserted state already exists in an extracted list.

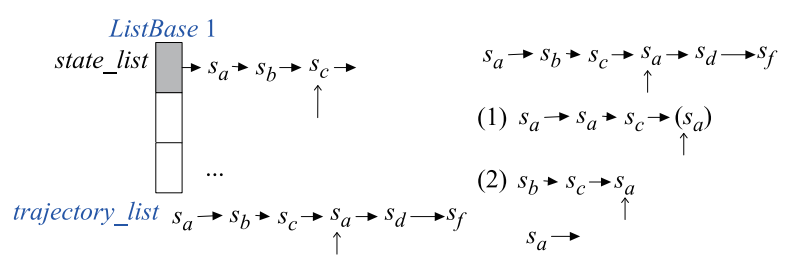

(a) Before inserting

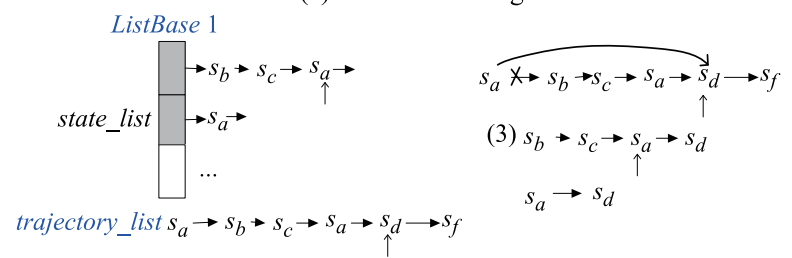

(b) Inserting

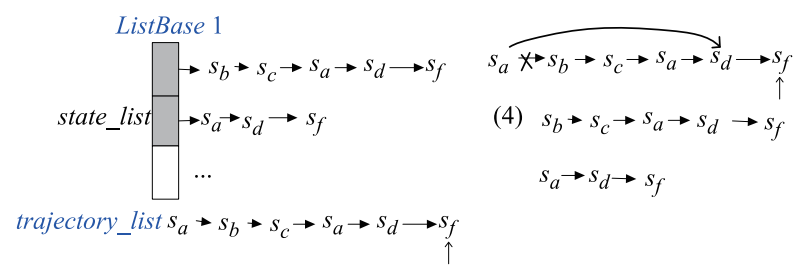

(c) After inserting

Fig. 6 Inserted state existing in an extracted list

The current state $s$ of an agent is usually inserted in the rear of the extracted list state_list. If the state $s$ exists in the extracted list, as shown in Fig. 6(a), a current state $s_{a}$ has already appeared in a state list earlier. The state $s_{a}$ is inserted at the rear of state_list. Apparently, this inserting position is nearer to the goal state than the position of the former state $s_{a}$. If the $Q$ value of the first $s_{a}$ is less than the second $s_{a}$, we add a list from the first $s_{a}$ to the next state of second $s_{a}$, i.e. $s_{d}$, otherwise insert $s_{a}$ in the list rear. This 
list means that transferring from $s_{a}$ to $s_{d}$ directly is better. It is not necessary to keep the sub-list from the first $s_{a}$ to the second $s_{a}$. Therefore, we just need to copy the sublist from the next state of $s_{a}$ to the rear of the state_list, act as a new extracted list with $s_{a}$, and add this new list into the shared list set ListBase1. The process is shown as Fig. 6(b). The state with arrow denotes the current state to be processed.

(ii) The state inserted already exist in the shared list set

The inserted state $s$ has appeared in one or several lists of the shared list set ListBase1, and the position of this state $s$ is closer to the goal state than the available state $s$. For example, the current state $s_{b}$ in Fig. 7(a) has already appeared in a state list of ListBase1. We update the shared lists as Fig. 7(b).
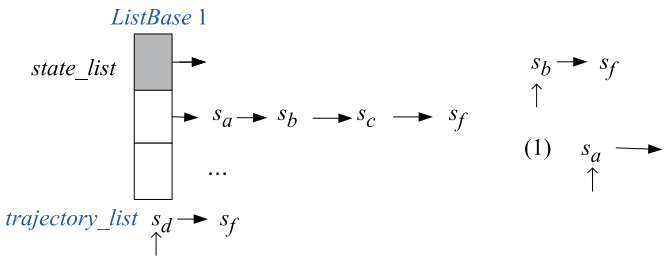

(a) Before inserting
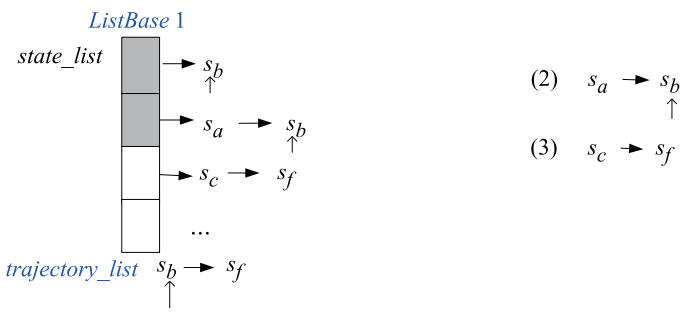

(3) $s_{c} \rightarrow s_{f}$

(b) Inserting
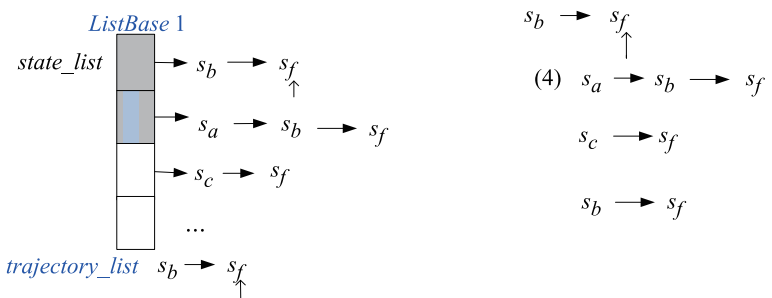

(c) After inserting

Fig. 7 Inserted state existing in the set of shared lists

Firstly, we seek the first state $s^{\prime}$ of the sub-lists of the extracted list with the state $s$ and copy a sub-list from $s^{\prime}$ to the state $s$ as an extracted list. Secondly, copy from the next state of the state $s$ to the rear, create a new current state list as Fig. 7(b). Thirdly, create a new extracted list and insert state $s$ as its rear as shown in Fig. 7(c), which is the first state also.

These extracted state lists form experience knowledge. Agents can share the knowledge with each other. The process of extracting state list is given in the algorithm of extracting state lists (ESL).
Algorithm ESL: Extracting state lists

Initialize: ListBase 1 is set to a null set; state_list includes a null list; $p$ is a pointer and is set to 0 .

Input: trajectory_list is a state trajectory of an agent, which includes the states an agent passed. $s=$ trajectory_list $[p]$.

Output: ListBase 1

(i) If a state $s$ already exists in an extracted list as shown in Fig. 6,

Update the extracted list by copying the sub-list from the second state to the end;

Insert the state $s$ at the end of a current state list;

Create a new extracted list with the state $s$;

(ii) If a state $s$ already exists in a list of the shared list set ListBase 1, as shown in Fig. 7;

Seek the first state $s^{\prime}$ of sub-lists with state $s$ in the extracted list;

Copy a sub-list from state $s^{\prime}$ to $s$ as an extracted list;

Copy from the next state after state $s$ to the rear as a new current, and insert it in the shared list set;

Create a new extracted list with the state $s$ as a list head;

(iii) If a state $s$ does not exist in any list of ListBase 1

Insert the state $s$ into state _list.

(iv) $p=p+1$;

If trajectory_list $[p] \neq$ null

$s=$ trajectory_list $[p]$;

go to step (i);

(v) Return ListBase 1.

As all states of trajectory_list are processed one by one, a shared state list, state_list, is built and added to the set ListBase 1.

\section{Optimal action set}

Agents in the same team or in different task teams can share the state list set ListBase 1 which is an experience knowledge base. Therefore, the experience knowledge can be propagated among all teams.

(i) Optimal action set

The optimal action for a state may be multiple during the learning process, so a set $U A$ is used to save optimal actions. If $a^{*}$ is the optimal action of a state $s$, then $a^{*} \in U A(s)$. The optimal actions can be calculated by the extracted state lists. For example, according to Theorem 1, the optimal action of the state $s_{i}$ is $a_{t+k}$ in Fig. 2, which is the action of the state $s_{i}$ near the final state. The optimal action of each state is added into the set $U A$ until the current state.

(ii) Experience propagation

If $a$ is the action an agent takes, an agent moves from the state $s$ to the state $s^{\prime}$ by executing the action $a$. We use the 
state list set ListBase 1 to refine the $Q$ values of the states affected by the set $U A$. The $Q$ value is updated as

$$
\boldsymbol{Q}(s, a)=\left\{\begin{array}{l}
r+\gamma \max _{a^{\prime}} \boldsymbol{Q}\left(s^{\prime}, a^{\prime}\right), \quad a \in U A(s) \\
\boldsymbol{Q}(s, a), \quad \text { otherwise }
\end{array}\right.
$$

where $r$ is an instant reward, and $\gamma$ is a discount factor.

The second refinement based on the optimal action set is given as the algorithm of experience propagation based on optimal action set (EPOOAS).

Algorithm EPOOAS( $s)$ : Experience propagation based on optimal action set

$U A \leftarrow$ null;

//Calculate the optimal action set $U A$ by using ListBase2; For $\forall p \in$ ListBase 2 ending at the state $s$

$\{$ For $i=2$ to length $(p)$

\{Getting the action $a$ from the state $p[i-1]$ to $p[i]$; $U A(p[i-1]) \leftarrow a\} ;$

For each non-final state $s$ of the list $p, \boldsymbol{Q}(s, a)$ is refined as (8) according to $U A(s)\}$.

\section{Reinforcement learning based on experien- ce sharing}

The action selection policy, $\pi(s)$, makes a decision based on the value of a value function or $\boldsymbol{Q}(s, a)$. When the selected action is the optimal action of a state, it is necessary to be reinforced again. The refinement of the $Q$ value follows the standard $Q$ learning. The second refinement of (8) is based on the experience knowledge. The algorithm that an agent extracts shared knowledge and adds it into the state lists base is shown as algorithm of reinforcement learning based on experience sharing (RLES).

Algorithm RLES: Reinforcement learning based on experience sharing

$s:$ a state;

$U A(s)$ : a null action set;

(i) Select an action $a$ according to the $Q$ values and execute it;

Get a next state $s^{\prime}$ of the state $s$, an instant reward $r$;

(ii) Update $\boldsymbol{Q}(s, a)$ as follows:

$$
\boldsymbol{Q}(s, a) \leftarrow \boldsymbol{Q}(s, a)+\alpha\left[r+\gamma \max _{a^{\prime}} \boldsymbol{Q}\left(s^{\prime}, a^{\prime}\right)-\boldsymbol{Q}(s, a)\right]
$$

Insert state $s^{\prime}$ in the state list trajectory_list;

(iii) Obtain a shared list set ListBase 1 by using the algorithm ESL;

(iv) Add the lists in ListBase 1 but not in ListBase 2 to ListBase2;

(v) Call the algorithm $\operatorname{EPOOAS}(s)$;

(vi) $s \leftarrow s^{\prime}$;

Go to step (i).
When an agent moves from a state $s$ to an other state $s^{\prime}$, the $Q$ value of the state $s$ is updated. The state $s^{\prime}$ is added into the state trajectory_list and then the shared state list ListBase 1 is computed by using this list. We check each list in ListBase 1 whether it has already existed in ListBase2. If not, add this state_list into the ListBase 2 which is a shared knowledge base. In step (v) of the algorithm, the optimal action set $U A(s)$ is calculated based on shared state lists. If the selected action at the current state belongs to the optimal action set $U A(s)$, this action should be reinforced again. After the $Q$ value is updated in step (i), the actions to be selected, which belong to the optimal action set, are again refined by heuristic selection of actions.

\section{Experiments and analyses}

We design a competition game based on the pursuit problem. The game performs in a simulation environment which is a $15 \times 15$ discrete grid with or without obstacles. There are some predators and preys in the grid. All the predators and preys are described as agents. Each agent can move to one of its four neighbor cells or stay in its current position. The action set is $\{$ stop, up, down, left, right $\}$. Any two predators cannot stay in the same grid cell. When two predators collide, they are penalized. When an agent is surrounded by opponents, it will be killed. Each agent moves from its start position, and ends killing all opponents or being captured by opponents. Every one of predators and preys must avoid being surrounded by opponents. Predators always hope to reach the goal as soon as possible with few steps.

We design four pursuit experiments to prove that the $Q$ learning based on experience extracting is an effective algorithm.

(i) Experiment environment

In the experiments we assume that all agents are provided with global vision. The agents in different teams cannot communicate with each other. A prey is captured when surrounded by predators and vice versa. The parameters setting of four experiments are four predators and one prey, eight predators and two preys, respectively. The policy of the predators is $Q$ learning based on experience extracting and the policy of the prey is random action.

For the $Q$ learning the parameters are as follows: a predator receives a reward 1000 when it helps to capture a prey and a negative reward -1000 when it is captured by preys. In a learning step, when a predator is close to its goal, it gets a reward 5 as encouragement, otherwise, it will get a reward -5 as punishment. The discount factor $\gamma$ is 0.9. The learning factor $\alpha$ is reduced from the value 1 gradually. The maximal step on pursuit failures is 8000 . The policy of the prey is fixed. It moves to adjacent 
position with a probability of 0.6. The global task can be divided into some subtasks. The agents of a subtask have a same competitor. Here it is known as completing a task that predators capture all preys.

(ii) Results of experiments

We compare the RLES with other two algorithms by experiments. One is that agents in a team or group learn based on experience sharing of $Q$ learning, but do not use extracted state list (GESNS). Another is that each agent learns independently based on standard $Q$ learning.

We draw the figures by using the average value of per four steps. Only the results of first $100 \times 4$ iterations of 1000 iterations are given in Fig.8 and Fig. 9. The experiment results of four predators versus one prey are shown in Fig. 8. The experiment results are given in Fig.8(a) with obstacles, and in Fig.8(b) without obstacles. The experiment results of eight predators versus two preys with or without obstacle are shown in Fig. 9.

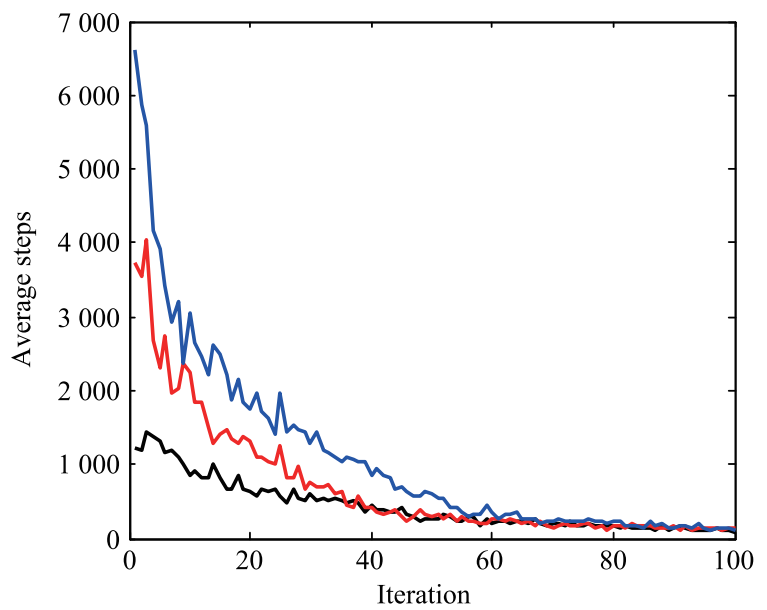

(a) With obstacles

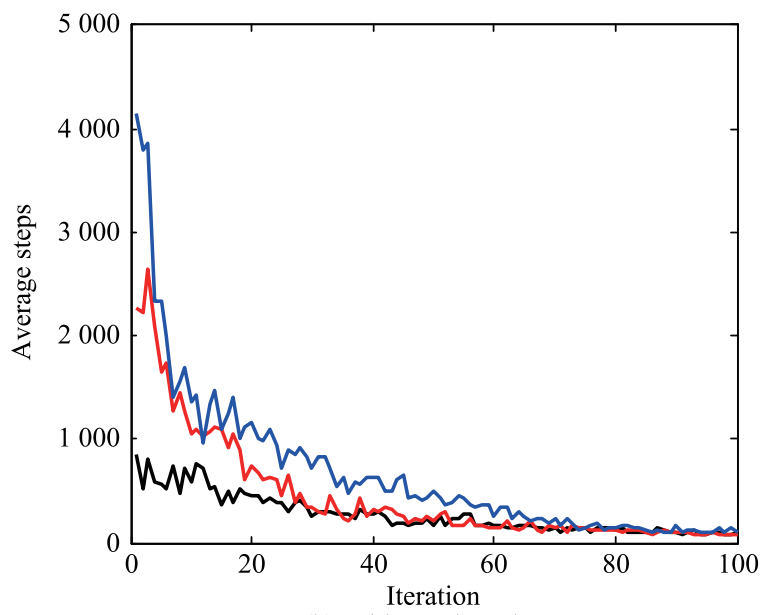

(b) Without obstacles

: RLES; : GESNS;

Fig. 8 Experiment results of four predators versus one prey

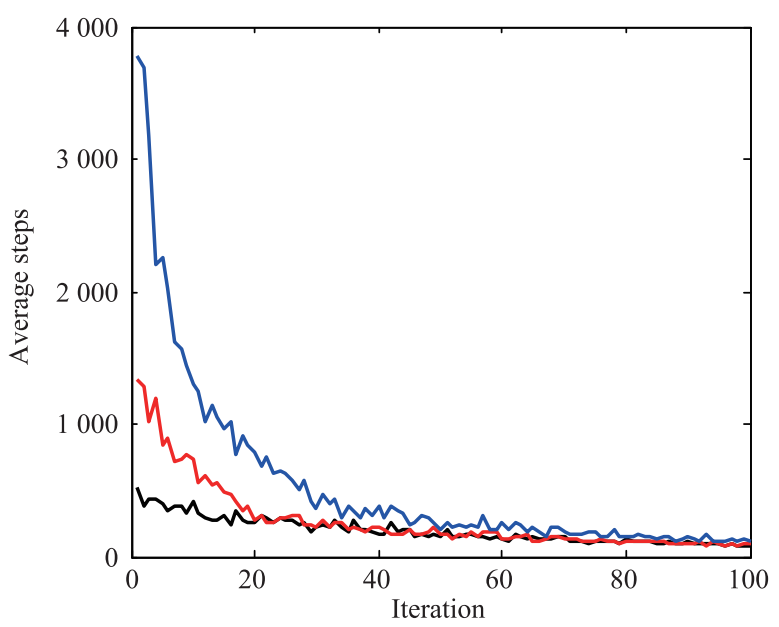

(a) With obstacles

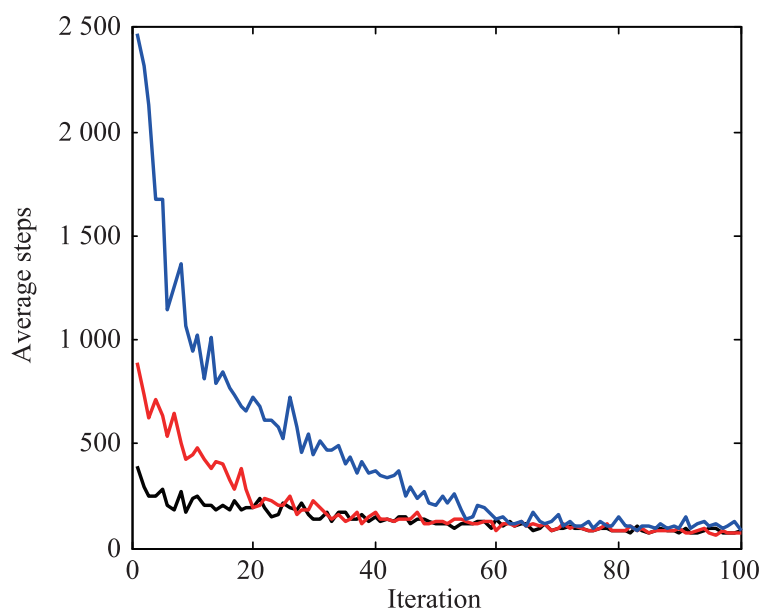

(b) Without obstacles

— : RLES; — : GESNS; — QL.

Fig. 9 Experiment results of Eight predators versus two preys

It is clear that the algorithms RLES and GESNS perform better than $Q$ learning according to Fig. 8 and Fig. 9, and the algorithm RLES has the best performance. The algorithm RLES can converge in fewer numbers of steps and with the fastest convergence rate.

When we compare the experiment results shown in Fig. 8(a), Fig. 9(a) with the results in Fig. 8(b), Fig. 9(b), respectively, we find that the agents without obstacles spend more time in catching preys by the three algorithms RLES, GESNS and $Q$ learning. The reason is that predator agents can surround preys with the assistance of obstacles. We find that the number of predators influence the performance of algorithms. Eight predators spend less time to reach a goal than four predators, as can be seen by comparing Fig. 8 with Fig. 9. When the number of agents is large, we can get more subtask groups. Each group can select more appropriate members. At the same time, for $Q$ learning with experience sharing, the more agents there 
are, the more experience can be shared.

\section{Conclusions}

In multi-agent reinforcement learning, to provide an effective interaction method for knowledge exchange and sharing among agents, we propose a state list extracting and sharing method based on a cooperative multi-agent reinforcement learning approach. This approach takes advantage of the state trajectory the agent wander in state space to compute the state lists to be shared, from an acyclic state paths analysis and extraction. It represents the state space knowledge the agents acquire from learning. Agents can share the state space knowledge with one another and propagate refined value functions to other states, even to those states they never reach in their episodes. Based on the state lists base, the value function of the cooperative multi-agent reinforcement learning will converge faster, and speeds up learning.

\section{References}

[1] P. C. Zhou, B. R. Hong, Q. C. Huang. A novel multi-agent reinforcement learning approach. Acta Electronica Sinica, 2006, 34(8): 1488-1491.

[2] J. G. Jiang, Z. P. Su, M. B. Qi, et al. Multi-task coalition parallel formation strategy based on reinforcement learning. Acta Automatica Sinica, 2008, 34(3): 349-352.

[3] M. Fang, F. C. A. Groen, H. Li. Dynamic partition of collaborative multiagent based on coordination trees. Proc. of the $12 \mathrm{th}$ International Conference on Intelligent Autonomous Systems, 2012.

[4] S. Abdallah, V. Lesser. Multiagent reinforcement learning and self-organization in a network of agents. Proc. of the 6th International Conference on Autonomous Agents and Multiagent Systems, 2007: 172-179.

[5] V. Conitzer, T. Sandholm. AWESOME: a general multiagent learning algorithm that converges in self-play and learns a best response against stationary opponents. Machine Learning, 2007, 67(1/2): 23-43.

[6] B. Banerjee, J. Peng. Generalized multiagent learning with performance bound. Autonomous Agents and Multiagent Systems, 2007, 15(3): 281-312.

[7] S. Kapetanakis, D. Kudenko. Reinforcement learning of coordination in heterogeneous cooperative multi-agent systems. Proc. of the 3rd Autonomous Agents and Multi-Agent Systems Conference, 2004: 1258-1259.

[8] L. Panait, S. Luke. Cooperative multi-agent Learning: the state of the art. Autonomous Agents and Multi-Agent Systems, 2005, 11(3): 387-434.

[9] M. C. Gifford, A. Agah. Sharing in teams of heterogeneous, collaborative learning agents. International Journal of Intelligent Systems, 2009, 24(2): 173-200.

[10] P. Hoen, E. D. de Jong. Evolutionary multi-agent systems.
Proc. of the 8th International Conference on Parallel Problem Solving from Nature, 2004: 872-881.

[11] G. Tesauro. Extending $Q$-learning to general adaptive multiagent systems. Advances in Neural Information Processing Systems, 2004, 16: 26-37.

[12] C. Zhang, V. R. Lesser, S. Abdallah. Self-organization for coordinating decentralized reinforcement learning. Proc. of the 9th International Conference on Autonomous Agents and Multiagent Systems, 2010: 739-746.

[13] M. Petrik, S. Zilberstein. Average-reward decentralized Markov decision processes. Proc. of the 20th International Joint Conference on Artificial Intelligence, 2007: 1997-2002.

[14] J. Li, Q. S. Pan, B. R. Hong. A new multi-agent reinforcement learning approach. Proc. of the IEEE International Conference on Information and Automation, 2010: 1667-1671.

[15] H. V. Seijen, S. Whiteson, H. V. Hasselt, et al. Exploiting bestmatch equations for effcient reinforcement learning. Journal of Machine Learning Research, 2011, 12(6): 2045-2094.

[16] J. Zhao, W. Y. Liu, J. Jian. State-Clusters shared cooperative multi-agent reinforcement learning. Proc. of the 7th Asian Control Conference, 2009: 129-135.

[17] T. G. Dietterich. Hierarchical reinforcement learning with the MAXQ value function decomposition. Artificial Intelligence Research, 2000, 13: 227-303.

[18] M. E. Taylor, G. Kuhlmann, P. Stone. Autonomous transfer for reinforcement learning. Proc. of the 7th International Joint Conference on Autonomous Agents and Multi-agent Systems, 2008: 283-290.

[19] M. E. Taylor, P. Stone, Y. Liu. Transfer learning via inter-task mappings for temporal difference learning. Machine Learning Research, 2007, 8(1): 2125-2167.

[20] L. Torrey, J. Shavlik, T. Walker, et al. Skill acquisition via transfer learning and advice taking. Proc. of the 17th European Conference on Machine Learning, 2005: 425-436.

\section{Biographies}

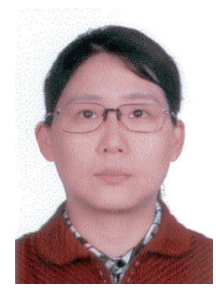

Min Fang was born in 1965. She received her B.S. degree in computer control, M.S. degree in computer software engineering and Ph.D. degree in computer application from Xidian University, Xi'an, China, in 1986, 1991 and 2004, respectively, where she is currently a professor. Her research interests include intelligent information process, multi-agent system and network technology.

E-mail: mfang@mail.xidian.edu.com

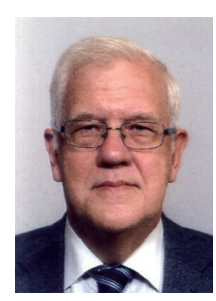

Frans C.A. Groen was born in 1947. He received his B.S., M.S. and Ph.D. degrees in applied physics from the Technical University of Delft, Netherlands. Since 1988 he has been a full professor at the University of Amsterdam. His research focuses on intelligent autonomous systems and multi-agent systems.

E-mail: F.C.A.Groen@uva.nl 